International Journal of Current Advanced Research

ISSN: O: 2319-6475, ISSN: P: 2319 - 6505, Impact Factor: SJIF: 5.995

Available Online at www.journalijcar.org

Volume 6; Issue 3; March 2017; Page No. 2539-2542

DOI: http://dx.doi.org/10.24327/ijcar.2017.2542.0045

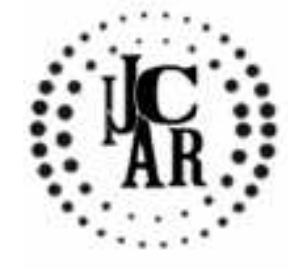

Research Article

\title{
NOISE REDUCTION BY OPTIMIZING THE AIRCRAFT ROUTE
}

\section{Kasturi E*and Prasanna Devi S}

Department of Computer Science \& Engineering, Apollo Engineering College, Chennai, India

\begin{tabular}{l}
\hline A R T I C L E I N F O \\
\hline Article History: \\
Received $18^{\text {th }}$ December, 2016 \\
Received in revised form $19^{\text {th }}$ January, 2017 \\
Accepted $4^{\text {th }}$ February, 2017 \\
Published online $28^{\text {th }}$ March, 2017 \\
\hline
\end{tabular}
\begin{abstract}
A B S T R A C T
Aircraft route optimization is key area in which the airlineindustry is focusing. The optimization of the route results in the reasonable profit along with the considerable aviation noise.Aviation noise can never be completely avoided around but it can be reduced to some extent.Thepaper proposes a comparison with the four algorithms which optimizes the route along with the decibel of noise produced in that course. The results show the reduction in aviation noise when the route is optimized and the best is also obtained.
\end{abstract}

Key words:

Aviation Noise, Bat Algorithm, Route

Optimization, Bcf Algorithm

Copyright $@ 2017$ Kasturi E and Prasanna Devi S. This is an open access article distributed under the Creative Commons Attribution License, which permits unrestricted use, distribution, and reproduction in any medium, provided the original work is properly cited.

\section{INTRODUCTION}

Airports are major centers of attraction in urban areas. Their impacts are many and usually include economic, environmental and land-use issues, all of which may affect the future development of the airport.One of the major problems affecting the people living and working around airport is noise due to aircraft operation. Accordingly, residential developments near noise-sensitive airport have generated all sorts of complaints and community actions aiming to reduce noise due to aircraft operation. Today's new generation aircraft are about $75 \%$ quieter than those built 40 years ago. However, the issue of concern for many people living in these areas is not due to the level of noise generated by individual aircraft, but rather the cumulative impact of a large number of over flights. They perceive that the periods of respite are rapidly disappearing and the noise events are becoming more frequent in the sensitive time periods such as evenings and nights.This change in the nature of the noise pattern would appear to be a significant factor in the widening geographic range of adverse community reaction to aircraft noise for the community residing in the proximity of airport globally.

*Corresponding author: Kasturi E

Department of Computer Science \& Engineering, Apollo

Engineering College, Chennai, India

\section{Domestic (INDIA) and International Scenario}

\section{Domestic Scenario}

The noise levels have been broadly classified under four categories by Ministry of Environment \& Forests vide The Noise Pollution (Regulation and Control) Rules, 2000:

Table 1 Domestic Noise pollution category

\begin{tabular}{cccc}
\hline $\begin{array}{c}\text { Sl. } \\
\text { No. }\end{array}$ & Category & \multicolumn{2}{c}{$\begin{array}{c}\text { Limits in dB(A) Leq } \\
\text { Day Time }\end{array}$} \\
\hline 1. & Industrial Time \\
2. & Commercial area & 75 & 70 \\
3. & Residential area & 55 & 55 \\
4. & Silence area & 50 & 45 \\
\hline
\end{tabular}

An airport though falls under industrial area category however, the noise levels are expected to be higher in the vicinity of the airport due to frequent aircraft movements. Most of the busiest airport world over has defined a separate area known as "Airport Vicinity Zone" wherein the ambient noise levels are expected to be higher than any other category of noise levels due to arriving and departing aircraft. Considering the fact that airport are usually located within the limits of large urban areas, in order to minimize the adverse impacts of its operation, it is necessary to organize the airport and surrounding areas through the development and adoption of a set of plans that govern urban planning and management with respect to the airport. It is important to notice that each airport is different in its operational characteristics, its social, economic and political situations, as well as in the type of landuse in its surrounding areas. All of these factors must be taken into account when planning land use in the vicinity of airport. 
However, in absence on a dedicated Airport zone and improper land planning in the vicinity of airport in India, the community residing in these areas are facing day to day problem due to large volume of aircraft operation. At one hand, the growth in aviation sector and airport developments is favorable to have growth in national Gross Domestic Product (GDP) but at the other hand, it leads to large annoyance to the communities residing in the vicinity of the airport.

\section{International Scenario}

Several international standards and methods to measure sound already exist to determine noise limits during the day/night due to aircraft operation in the airport vicinity. All the above mentioned standards are developed taking into account the number of aircraft movements, type of aircraft operating at that airport, geographical location of the airport, etc Some airport also provide Noise Limits for aircraft, essentially to control engine noise during approach/take off from runways. Some of the airport with noise limits are listed in table2

Table 2 List of airport with noise limits

\begin{tabular}{cccc}
\hline \multicolumn{4}{c}{ Noise levels at International Airport } \\
\hline Sl. No. & International Airport & Country & $\begin{array}{c}\text { Permissible Noise } \\
\text { Limits } \\
\text { (peak values) }\end{array}$ \\
\hline 1. & JFK- New York & USA & 99dB (A) \\
2. & Midway- Chicago & USA & No limits \\
3. & Rio de Janerio & Brazil & $118.4 \mathrm{~dB}(\mathrm{~A})$ \\
4. & Brussels & Belgium & $100 \mathrm{~dB}(\mathrm{~A})$ \\
5. & Rome & Italy & $103 \mathrm{~dB}(\mathrm{~A})$ \\
6. & Gatwick- London & UK & $94 \mathrm{~dB}(\mathrm{~A})$ \\
7. & Heathrow - London & UK & $94 \mathrm{~dB}$ (A) \\
8. & Stansted -London & UK & $94 \mathrm{~dB}(\mathrm{~A})$ \\
9. & Manchester & UK & $92 \mathrm{~dB}(\mathrm{~A})$ \\
10. & Hong Kong & Hong Kong & No Limits \\
11. & Melbourne & Australia & No Limits \\
12. & Osaka & Japan & $107 \mathrm{~dB}(\mathrm{~A})$ \\
13. & Kuala Lumpur & Indonesia & No Limits \\
14. & CDG - Paris & France & $104.5 \mathrm{~dB}(\mathrm{~A})$ \\
\hline
\end{tabular}

It is pertinent to mention that permissible noise limits at above mention airport varies primarily due to the difference in their topological locations. The airport situated in the northern hemisphere have lower noise limits than those located near the equator due to the fact that the air is more cold and dense which acts as a medium to absorb some noise.[11]In line with International Civil Aviation Organization's (ICAO) mandate to reduce or minimize aircraft noise through an established noise management programme, DGCA has initiated a proactive role to minimize the airport noise in whole. Besides reducing the noise at source, emphasis is being given to reduce the airport noise by sources other than aircraft also.[14] It has been observed that noise due to aircraft movement add to the problem of the community noise in the vicinity of the airport apart from airport noise.

\section{Assess the aircraft noise}

When assessing the environmental affect of aviation noise, the air transport method as a whole wishes to be regarded in view that overall noise and the reduction of the same is to be established.Explanations akin to plane utilization and routing grow to be very fundamental in terms of whole noise and operatingcosts.[12] This is a exchange from the historical plane design philosophy which makes a speciality of minimizing charges on a small set of missions.[13] There has been some latest work on the design of aircraft for single hub route networks, however it didn't include environmental performance as an objective or constraint [11].An method is provided in this paper that allows to optimize a set of a number of aircraft at a conceptual design stage for a detailed route network.[9] The targets of curiosity comprise the financial and noise performance of a fleet over the route network. There are a lot of choices to be made by way of the airlines to fulfill market demand. The most important picks are the size and performance of the aircraft themselves.[6] The route community overwhich the aircraft operate additionally has a gigantic impacton the monetary efficiency and total noise in the environment. In this study we considerboththe route network and the noise reduction which indicates that two objectives has to be satisfied.

\section{Bio -inspired Algorithms for optimization of air route}

We are opting for nature situated meta-heuristic algorithm considering, heuristics are in general main issue-elegant, in which we define an heuristics for a given problem to find the best solution. Meta-heuristics algorithms are challengeindependent methodologies that may be applied to a huge variety of issues for analysis among which the bio-inspired algorithms have now taken their chance to prove their efficiency. An heuristic can be like opting for a random aspect for pivoting in Quicksort. A meta-heuristic is aware of nothing about the situation it is going to be applied, it will possibly deal with functions as black bins. We can say that a heuristic exploits obstacle-elegant understanding to finda most finest or first-rate technique to an targeted predicament, while metaheuristics are like design patterns, general algorithmic recommendations, which will also be applied to a large variety of problems. On this learn, the route profitability is optimized making use of bio-inspired algorithms like Firefly algorithm (FA), Bat algorithm (BA) and Cuckoo search algorithm (CSA), hybrid approach (BCF).[4,7] Dynamic Programming (DP) utilizing PL/SQLis used to search out the expected price of every route generated via FA, BA and CSA. Results: the target is to scale back the total expected price or maximize profit per airliner per route. The health worth of a airline and route is calculated utilizing DP. In the proposed model, we're making use of three algorithms wherein the initial particles are generated, centered on Nearest Neighbor Heuristic (NNH) which deals with the airliners. The algorithm is appliedutilizing PL/SQL and tested with issues having unique number of aviation knowledge set fromAustralian transportation from the 12 months Jan 2009 to Nov 2014. The outcome got are aggressive and confirmed some large growth over revenue, in phrases of execution time and memory usage as good.

\section{Analysis of Algorithms in the given data set}

The proposed multi-purpose BCF, firefly, bat and cuckoo search is implemented in PL/SQL to performroute profitability analysis on airline information set gathered from Australian aviation data. Firstly we have now verified thealgorithm effects for these three algorithms utilising aviation data for November 2014 which has 124 records along with 53 Australian ports and 572009 to November 2014 that contains 30,000 files of exact aviation ports which is the sample data set.[4]The time series plot for each algorithm along with the actual data is given in figure 7 , figure 8 , figure 9 , figure 10 , figure 11 , figure 12 . The results shown a great difference in the performance overseas ports. We have now carried out giant 
Table 3 comparison of the algorithms over the parameters

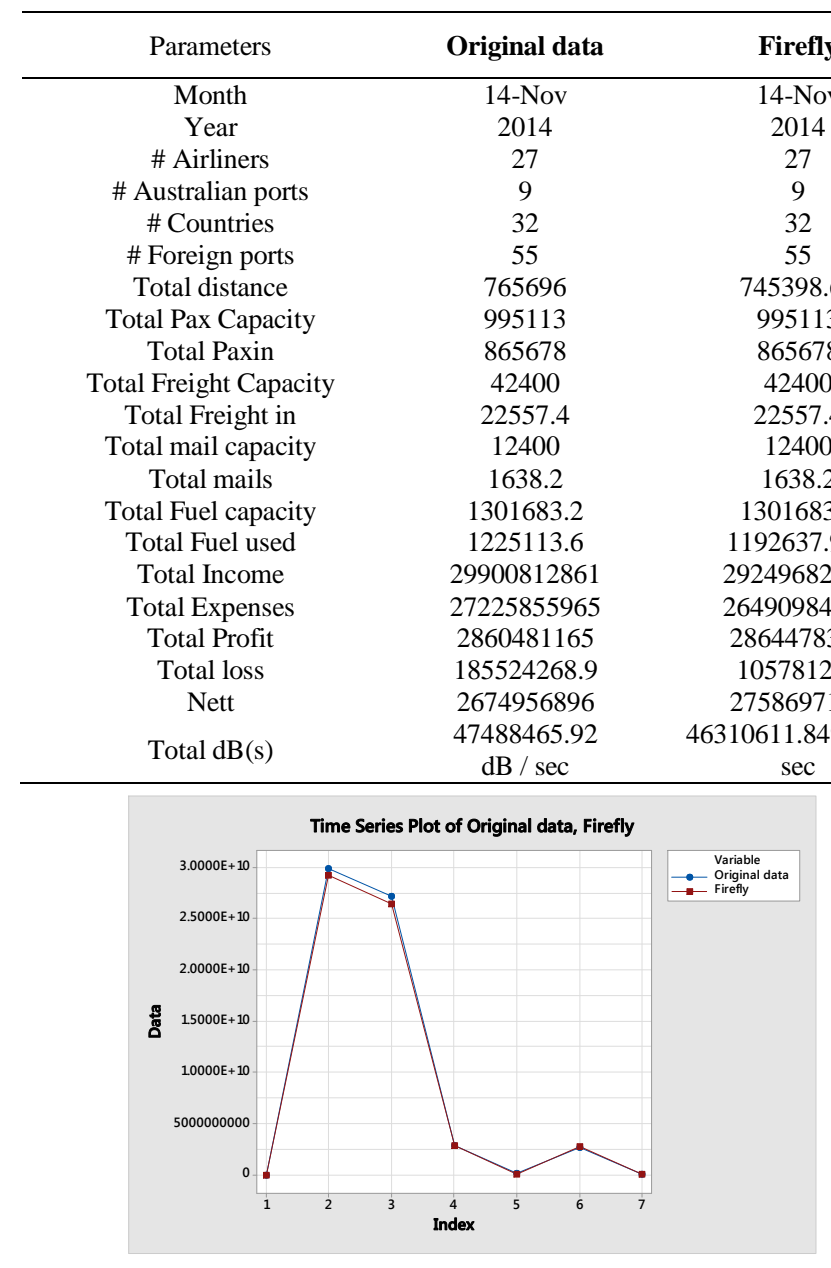

Figure 7

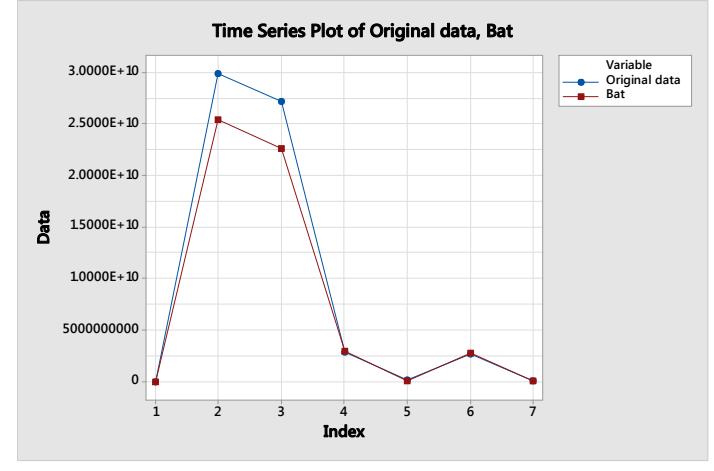

Figure 9

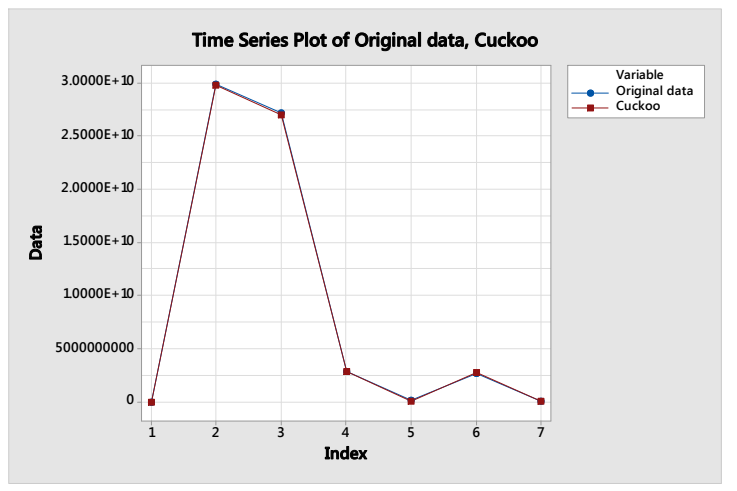

Figure 11

\begin{tabular}{ccc} 
Bat & Cuckoo & $\begin{array}{c}\text { Hybrid } \\
\text { Approach BCF }\end{array}$ \\
\hline $14-$ Nov & $14-$ Nov & $14-$ Nov \\
2014 & 2014 & 2014 \\
27 & 27 & 27 \\
9 & 9 & 9 \\
32 & 32 & 32 \\
55 & 55 & 55 \\
646873 & 712188 & 765696 \\
995113 & 995113 & 995113 \\
865678 & 865678 & 865678 \\
42400 & 42400 & 42400 \\
22557.4 & 22557.4 & 22557.4 \\
12400 & 12400 & 12400 \\
1638.2 & 1638.2 & 1638.2 \\
1301683.2 & 1301683.2 & 1301683.2 \\
1034996.8 & 1139500.8 & 1225113.6 \\
25461304048 & 29797861751 & 30200675073 \\
22657951619 & 27028323820 & 27058044202 \\
2918265155 & 2865026062 & 3264923343 \\
114912726 & 95488131 & 122292472 \\
2803352429 & 2769537931 & 3142630871 \\
$40189344 . .8$ & 44169899.76 & 47488465.92 \\
dB /Sec & dB / sec & dB / sec \\
\hline & &
\end{tabular}

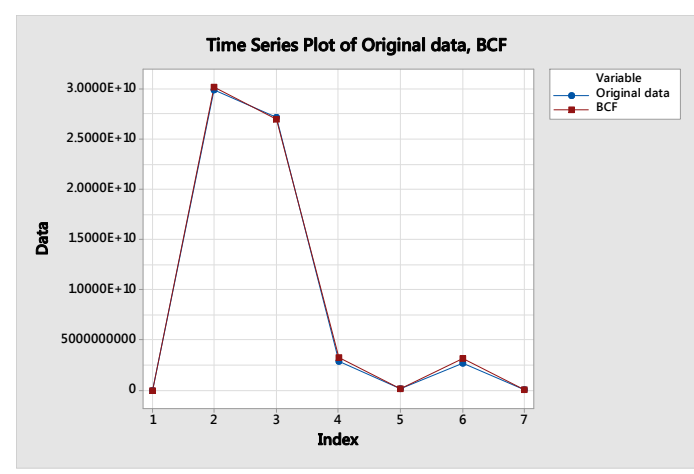

Figure 8

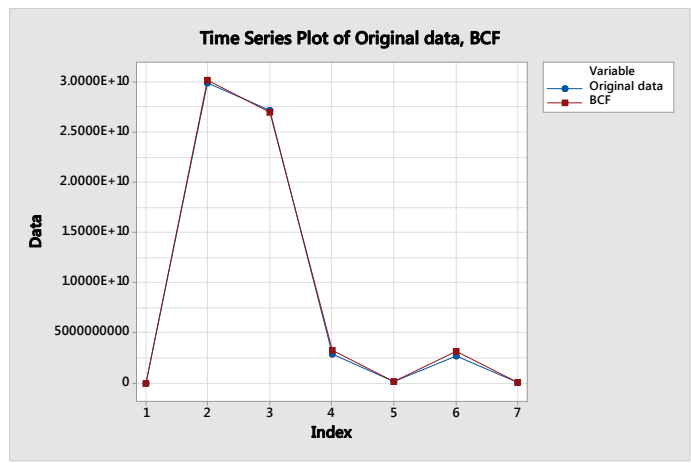

Figure 10

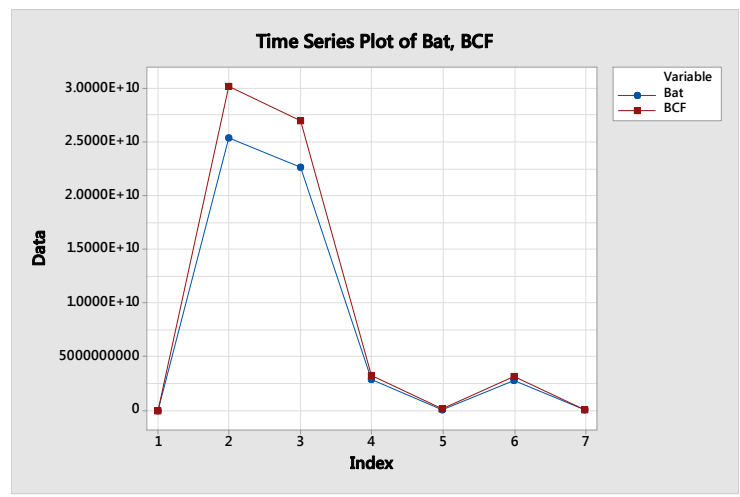

Figure 12 
data analysis on aviation knowledge fromJanuary .The performance of each algorithm over the original data is displayed in the table 3.

\section{CONCLUSIONS}

The data set when run in the algorithms got to show more advantageous results more than expected. All the algorithms performed well but the best is the bat and the BCF algorithm in which the profit is increased by optimizing the routes. As per the objective the bat algorithm shows considerable change in the noise level in the atmosphere triggered by the aircraft during the flight.And there is $8.39 \%$ reduction when compared to the $\mathrm{BCF}$ algorithm which counts really great for the noise in the atmosphere is reduced considerably by optimizing route of the aircraft.The matrix plot for all the algorithms over the original or the actual data is given in figure 6.The hybrid BCF algorithm integrated the route seat allotment thereby increasing the net amount which is far more than the other algorithms. The performance of the Bat and the $\mathrm{BCF}$ algorithm is compared in the figure4, figure5.The integration of the hybrid algorithm with the other algorithms may be considered in future so as to reduce the noise level also simultaneously.

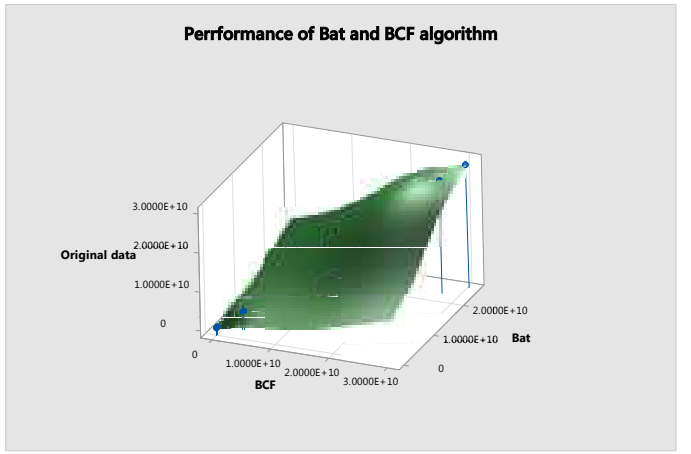

Figure 4

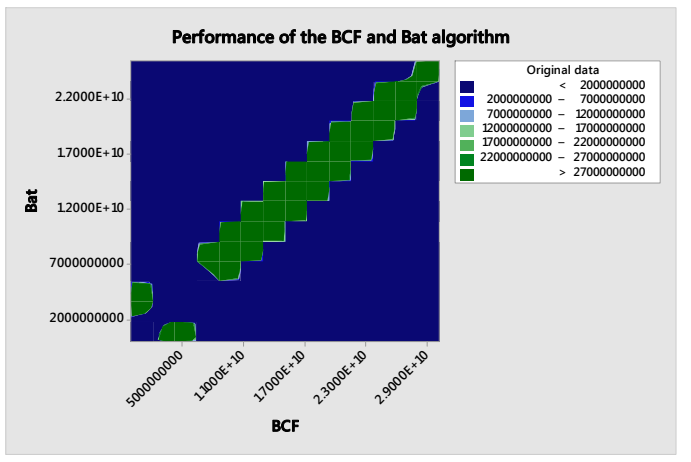

Figure 5

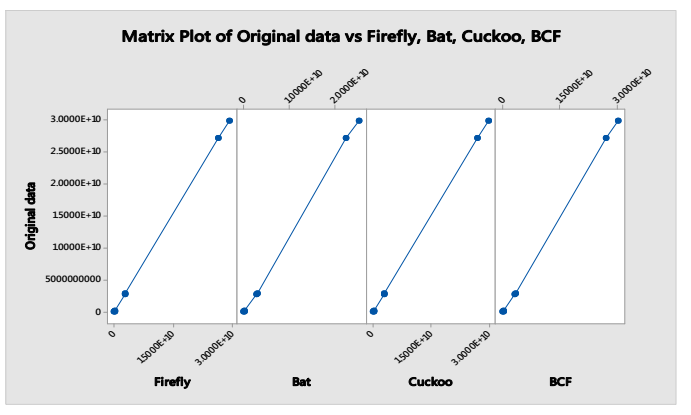

Figure 6

\section{Acknowledgements}

Australian government - International airline activity10for the Data set Big Data Analytics lab, Apollo Engineering College, Chennai, Tamil Nadu, India for the Lab

\section{References}

1. Swift, H., "A Review of the Literature Related to Potential Health effects of Aircraft Noise," PARTNER Project 19 Final Report, 2010.

2. Sizov, N., “Aviation Noise Impacts Research Roadmap 2011,” Report No. DOT/FAA/AEE/2011-07, 2011.

3. Cho, H., Azzam, M., Hansman, R. J., and Jensen, L. L., "Noise Analysis and Negotiation Tool for Terminal RNPProcedure Design," 2013 Aviation Technology, Integration, and Operations Conference, Los Angeles, CA, 2013

4. Kasturi E, Prasanna Devi S, Vinu Kiran S, Manivannan S (2016), Airline Route profitability analysis and Optimization using BIG DATA analytics on aviation data sets under heuristic techniques, Procedia Computer Science $87: 86$ - 92

5. 11SAE, "Procedure for the Calculation of Airplane Noise in the Vicinity of Airports," 1986.

6. Sizov, N., “Aviation Noise Impacts Research Roadmap 2011," Report No. DOT/FAA/AEE/2011-07, 2011.

7. E. Kasturi, Dr.S. Prasanna Devi, S. Vinu Kiran, (2015), Airline route profitability analysis on Bigdata using Firefly AlgorithmAust. J. Basic \& Appl. Sci., 9 (10) pp. 313-320

8. Fidell S, Tabachnick B, MestreV,Fidell L,(2013),Aircraft noise-induced awakenings are more reasonably predicted from relative than from absolute sound exposure levels, J AcoustSoc Am, Nov;134(5):3645-53.

9. Boeker, E., Dinges, E., He, B., Fleming, G., Roof, C., Gerbi, P., Rapoza, A., and Hemann, J., "Integrated Noise Model (INM) Version 7.0 Technical Manual," 2008.

10. https://bitre.gov.au/publications/ongoing/international_ airline_activity-time_series.aspx

11. Berglund B, Lindvall $\mathrm{T}$, eds. Community noise. Document prepared for theWorld Health Organisation. Archives of the Centre for Sensory Research1995;2:1195.

12. Heblij, S. J., and Wijnen, R. A. A., "Development of a runway allocation optimisation model for airport strategic planning," Transportation Planning and Technology, 31(2), 2008, pp. 201-214.

13. Braakenburg, M.L., "Development of a multi-event optimization tool for noise optimized arrival route design,"MSc thesis, TU Delft, November 2010.

14. Torres, R., Chaptal, J, Bès, C., and Hiriart-Urruty, J-B., "Optimal, Environmentally-Friendly Departure Procedures for Civil Aircraft," Journal of Aircraft, Vol 48, No 1, 2011, pp. 11-22 